\title{
Unilateral double testicular arteries surround renal vein
}

\begin{abstract}
Normally, male's testicular arteries are originating from the abdominal aorta. In this case, we noted that testicular artery variations are related to renal and/or aorta arteries. The aim of this presentation was to evaluate the testicular arteries in male's reproductive system and their contribution with renal function in some disorders. During routine dissection of abdomen's retroperitoneal region, in a 60-year-old male cadaver, we observed double variant testicular arteries with circle loop around left renal vein. Anatomical variations of testicular and renal vessels must be considered to solve male genital, and/or renal disorders.
\end{abstract}

Keywords: double testicular arteries, renal vein, artery-vein anastomosis
Volume 6 Issue 5 - 2019

\author{
Ahmad Tamjidipour, Asghar Rajabzadeh \\ Department of Anatomy, Lorestan University of Medical \\ Sciences, Iran
}

Correspondence: Asghar Rajabzadeh, Department of Anatomy, Lorestan University of Medical Sciences, Faculty of Medicine, Khorramabad, Iran, Email Da.rajabzadeh@yahoo.com

Received: June 16,2019 | Published: September II, 2019

\section{Introduction}

Bilateral testicular arteries (TAs) are the ventral subdivisions of abdominal aorta, mostly separated at the lower starting point of the renal arteries, and obliquely descended on the psoas major muscles. In the retroperitoneal region of abdomen, they run on the superior aperture of pelvis and enter into the spermatic cords in the inguinal canal. Clinical features and the awareness of TAs variants are associated with solving such a comprehensive issue of fertility and varicocele. ${ }^{1}$ Mostly some reports showed that variant testicular artery aroused from renal arteries or with double source of origin from aorta, frequently took place on the right side..$^{2,3}$ In our case report, we observed double TAs are originated from aorta in addition to testicular (artery)-renal (vein) anastomosis on the left side.

\section{Presentation}

In our anatomy department, a 60-year-old male cadaver fixed with formalin was used for the research and educational course. After one year, during routine dissection of posterior abdominal wall (level L1L2), we noticed that an artery with double origin arised from the left side of aorta (Figure 1). Two arteries were joined together under the renal vein, after forming loop around it. In fact, it seems that ascending branch of lower testicular artery was linked to renal vein (Figure 1). The upper testicular artery passes posteriorly to the renal vein and joins to the lower artery (Figure 2). This position of TAs displays an abnormal arterial pressure on renal vein. In this case, a significant point was hypertension of the left renal vein compared to the same vein on the right side. In the similar condition, this pressure can affect drainage of renal venous, and renal hypertension. ${ }^{1}$ Recorded figures of vessels morphology was obtained by high quality camera.

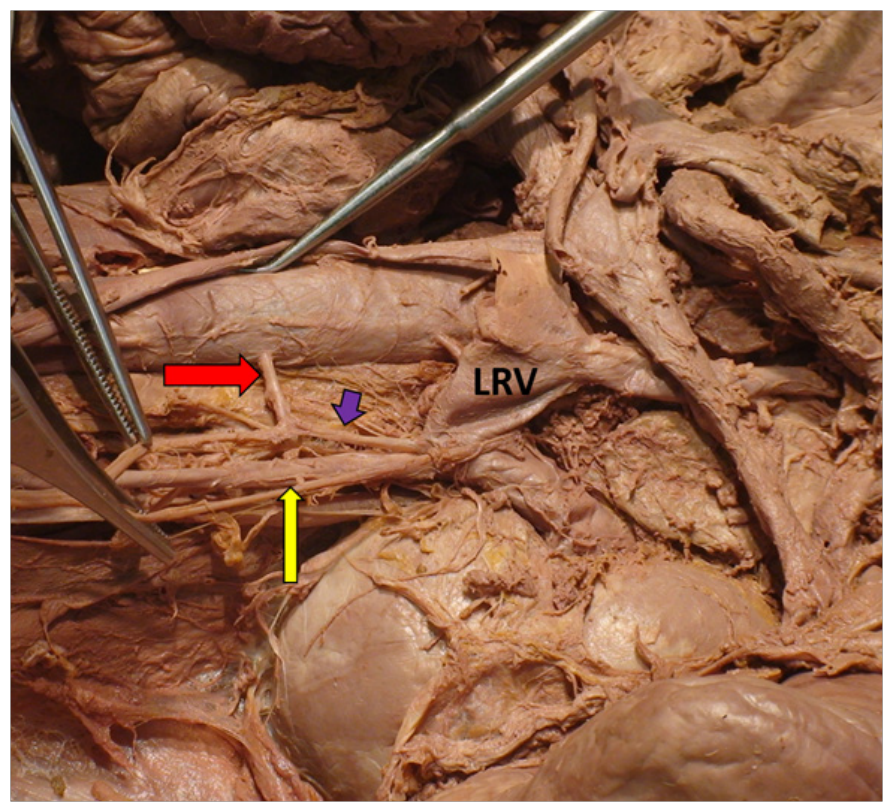

Figure I LRV, left renal vein; bold arrow, lower originated left testicular artery from aorta; thin arrow, descending part of highest testicular artery after rotated behind left renal vein; short arrow, ascending part of lower testicular artery that anastomosis with left renal vein. 


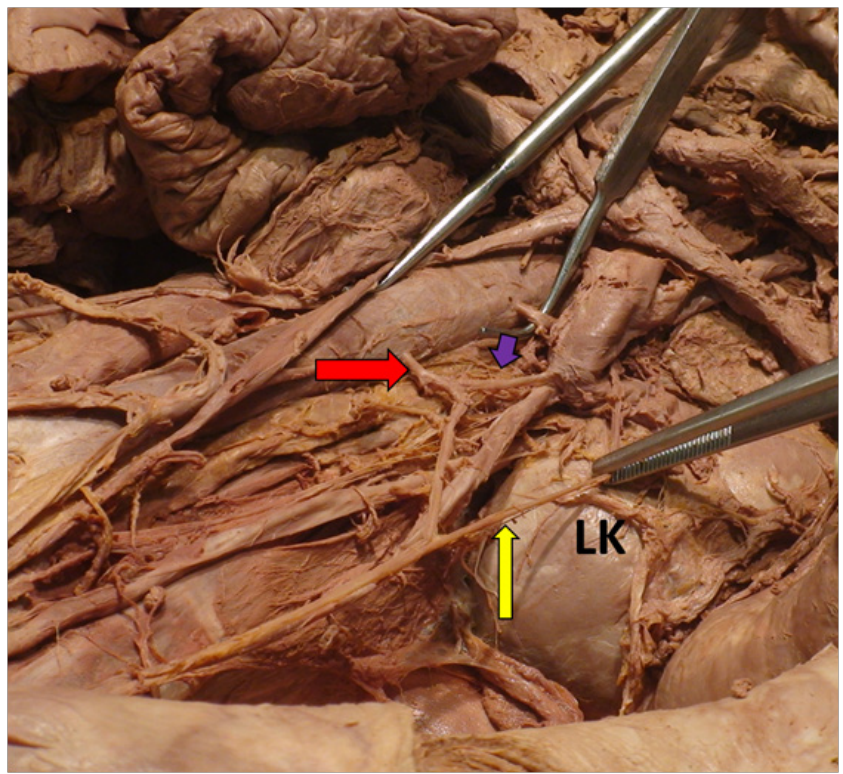

Figure 2 LK, left Kidney; bold arrow, lower originated left testicular artery from aorta; thin arrow, descending part of highest testicular artery after rotated behind left renal vein; short arrow, ascending part of Lower testicular artery that anastomosis with left renal vein.

\section{Discussion}

The variation of male's testicular arteries is may be considering due to important correlation with clinical complications, especially fertility or surgical performance in the retroperitoneal region related to kidneys. In this setting, anatomical information can respond to the most clinical questions in the male reproductive problems, as well as, nephrectomy and renal transplantation. ${ }^{4}$ Abnormal pressure of testicular vessels on renal vein may be effect on function, and choosing suitable surgery protocol of kidneys. ${ }^{5}$ The presence of some variance is critical when they reduce the blood supply to sensitivity organs as testis or kidneys. Although there are several reports on variant testicular arteries, most of them showed unilateral or bilateral double arteries. ${ }^{1}$ In this report, we also showed a variant case concerning to testicular artery that unusually the renal vein was surrounded by their branches, and likely abnormal anastomosis among artery and vein. Since 1928, reports of cadaver and surgical cases showed that variant TAs has been occurred on both right and left sides, with high contribution of right testicular artery. ${ }^{6-9}$ Also this is possible that these arteries originate from suprarenal artery which it only reported in $2012 .{ }^{10}$ Conclusively, our report presents a unique case of TAs variants concerning their source of origin and exclusively pressure from double testicular arteries onto hyper tensioned renal vein. Supplementary examinations such as radiological approaches, Doppler ultrasound or arteriography may be contributing to appropriate understanding of the gonadal and/ or retroperitoneal vessels.

\section{Conclusion}

Based on origination of testicular artery and its routes at the anatomical position, this point can be express that information of these vessels and their variations will make the best strategy of diagnosis in the male's genital disorders.

\section{Acknowledgments}

None.

\section{Conflicts of interest}

The authors declare there is no conflict of interest.

\section{References}

1. Paraskevas GK, Nastis K, Nitsa Z, et al. Bilateral double testicular arteries: a case report and review of the literature. Folia Morphol (Warsz). 2014;73(3):383-388.

2. Cicekcibasi AE, Salbacak A, Seker M, et al. The origin of gonadal arteries in human fetuses: anatomical variations. Ann Anat. 2002;184(3):275279.

3. Singh R, Jaiswal A, Shamal SN, et al. Variation in the origin of the testicular arteries and drainage of the right testicular vein. Int J Morphol. 2011;29(2):614-616.

4. Sarita S, Sridhar VK, Venkata RV, et al. Bilateral variant testicular arteries with double renal arteries. Cases J. 2009;2(1):114.

5. Dhar P, Lal K. Main and accessory renal arteries-A morphological study. Ital J Anat Embryol. 2005;110(2):101-110.

6. Jyothsna P, Mohandas Rao KG, Somayajii SN, et al. Multiple vascular anomalies involving testicular, suprarenal arteries and lumbar vein. $N$ Am J Med Sci. 2012;4(3):154-156.

7. Kayalvizhi I, Monisha B, Usha D. Accessory left testicular artery in association with double renal veins. Folia Morphol. 2011;70(4):309311 .

8. Loukas M, Stewart D. A case of an accessory testicular artery. Folia Morphol. 2004;63(3):355-357.

9. Pia MM, Vadgaonkar R, Rai R Nayak SR, et al. A cadaveric study of the testicular artery in the south Indian population. Singapore Med J. 2008;49(7):551-555.

10. Brohi RA, Sargon MF, Yener N. High origin and unusual suprarenal branch of a testicular artery. Surg Radiol Anat. 2001;23(3):207-208. 